\title{
A SUFFICIENT ARITHMETICAL CONDITION \\ FOR THE IDEAL CLASS GROUP OF AN IMAGINARY QUADRATIC FIELD TO BE CYCLIC
}

\author{
MAKOTO ISHIBASHI
}

(Communicated by William W. Adams)

\begin{abstract}
We give a sufficient arithmetical condition for the ideal class group of an imaginary quadratic field to be cyclic by using Ono's number.
\end{abstract}

\section{INTRODUCTION}

Let $m \leq-5$ be a squarefree integer. Put $K=Q(\sqrt{m})$. Euler observed that the polynomial $x^{2}+x+41$ is a prime for all integer $x, 0 \leq x \leq 39$, and Gauss showed that $Q(\sqrt{-163})=Q(\sqrt{1-4(41)})$ has a class number one. Put $f=(1-m) / 4$ if $m \equiv 1(\bmod 4)$ or $=-m$ otherwise, and define $P_{K}(x)=$ $x^{2}+x+f$ if $m \equiv 1(\bmod 4)$ or $=x^{2}+f$ otherwise. Then the FrobeniusRavinovitch theorem says that $P_{K}(x)$ is prime for $0 \leq x \leq f-2$ if and only if the class number $h_{K}$ of $K$ is one.

Sasaki generalized this in the following way. If $n=q_{1}^{e_{1}} \cdots q_{g}^{e_{g}}$ is the canonical factorization of $n$ into prime powers, write $L(n)=e_{1}+\cdots+e_{g}$ and define

$$
L_{K}=\max L\left(P_{K}(n)\right): 0 \leq n \leq f-2 .
$$

Sasaki [7] proved that $L_{K} \leq h_{K}$ and that $L_{K}=2$ if and only if $h_{K}=2$; the latter result is also implicit in a paper by Carlitz [2]. $L_{K}$ is known as Ono's number.

The aim of this paper is to prove the following generalization of Sasaki's result:

Theorem. Let $K=Q(\sqrt{m})$ be an imaginary quadratic field with class number $h_{K} \geq 3$. Write $h_{K}=h_{1} h_{2}$, where $h_{1}=\prod_{p \| h_{K}} p$ (i.e., the squarefree part of $\left.h_{k}\right)$. Suppose that $1 \leq h_{2}$ and put $q_{0}=\min \left\{q ;\right.$ rational prime and $\left.q \mid h_{2}\right\}$. If $L_{K} \geq q_{0}+h_{K} / q_{0}$, then the ideal class group $H_{K}$ of $K$ is cyclic.

\section{Czogala filtration}

For the preliminaries of our proof, let us recall the results of Czogala [3].

Received by the editors October 20, 1990 and, in revised form, March 11, 1991 and June 26, 1991; presented at the Japan Math. Soc. annual meeting, 1986, Kyoto.

1991 Mathematics Subject Classification. Primary 11 S99. 
Definition 1. Let $V$ be the family of all algebraic number fields. Take $F \in V$. For a fixed natural number $n \geq 2$, consider the property

if $a_{2} a_{2}=b_{1} \cdots b_{k}$ for irreducible elements $a_{1}, a_{2}, b_{1}, \ldots, b_{k}$

$(*)_{n} \quad\left(\right.$ for some $k \geq 2$ ) in $O_{F}$ (the ring of integers in $F$ ), then $k \leq n$.

Write $V_{n}=\left\{F \in V ; F\right.$ has the property $\left.(*)_{n}\right\}$.

Lemma 1. Let $(a)=P_{1} \ldots P_{n}$ be the decomposition into prime ideals $\left(P_{1}, \ldots\right.$, $P_{n}$ are not necessarily distinct, $\left.a \in O_{F}\right)$. Suppose that $P_{i}$ belongs to an ideal class $x_{i} \in H_{F} \quad(1 \leq i \leq n)$ and $x_{0}$ denotes the principal class of $H_{F}$. Then $a$ is an irreducible in $O_{F}$ if and only if $x_{i_{1}} \cdots x_{i_{m}} \neq x_{0}$ for every proper subset $\left\{i_{1}, \ldots, i_{m}\right\}$ of $\{1, \ldots, n\}$.

Lemma 2. Suppose that the principal ideal $(a)$ is expressed by the product of at most $n(\geq 2)$ prime ideals for every irreducible element $a \in O_{F}$. Then it is true that $F \in V_{n}$. Conversely, if $F \in V_{n}$ then the order of each class $x \in H_{F}$ is at most $n$.

Lemma 3. If $F \notin V_{n}$ then $n+1 \leq h_{F}$.

Proof. By Lemma 2, there exists an irreducible element $a \in O_{F}$ such that (a) $=P_{1} \ldots P_{n+1} A$ for some prime ideals $P_{1}, \ldots, P_{n+1}$ and for some ideal $A$ in $O_{F}$. Then it follows that $P_{1}, P_{1} P_{2}, P_{1} P_{2} P_{3}, \ldots, P_{1}, \ldots, P_{n+1}$ belong to distinct ideal classes because of Lemma 1 . Therefore we have $n+1 \leq$ $h_{F}$. Q.E.D.

Proposition 1. For every algebraic number field $F \notin V_{2}$, it is true that $n_{F} \leq h_{F}$, where $n_{F}=\min \left\{n \geq 2 ; F \in V_{n}\right\} \geq 3$.

Proof. As a consequence of Lemma 3, it follows that

$$
V=\bigcup_{n \geq 2} V_{n} \quad\left(V_{2} \subset V_{3} \subset \cdots \subset V_{n} \subset \cdots\right) .
$$

Thus $n_{F}$ is well defined and $F \notin V_{n_{F}-1}$. Hence we have $n_{F} \leq h_{F}$. Q.E.D.

\section{DAVENPORT'S CONSTANT}

Let us recall two definitions of Davenport's constant for a finite abelian (additive) group $H$ with order \# $(H)$. Define $u(H)$ as the minimal natural number $u$ such that for any $u$ elements $g_{1}, \ldots, g_{u}$ (they need not be distinct) in $H$ there exists a nonempty proper subset $\left\{i_{1}, \ldots, i_{m}\right\}$ of $\{1, \ldots, u\}$ with vanishing sum (i.e., $g_{i_{1}}+\cdots+g_{i_{m}}=0$ ). Define $a(H)$ as the maximal length of a sequence $\left\{g_{1}, \ldots, g_{a}\right\}$ of elements (not necessarily distinct) in $H$ such that $g_{1}+\cdots+g_{a}=0$ that contains no nonempty proper subsequence with vanishing sum. Then one can easily see that $u(H)=a(H) \leq \#(H)$. Especially, for every cyclic group $C_{n}$ with order $n$, we have $u\left(C_{n}\right)=a\left(C_{n}\right)=n$.

Lemma 4. Suppose that $H \cong C_{b_{1}} \oplus \cdots \oplus C_{b_{r}}$ and $b_{i} \mid b_{i+1} \quad(1 \leq i \leq r-1)$. Then it is true that $u(H) \leq b_{r}\left(1+\log \left(h / b_{r}\right)\right)$, where $h=\#(H)=b_{1} \cdots b_{r}$. Especially, if $r=2$, then $u(H)=b_{1}+b_{2}-1$.

Proof. See Theorem 7.1 in [1] and Corollary 1.1 in [5]. Q.E.D. 
Lemma 5. If $H$ is a noncyclic finite abelian group then $u(H) \leq \#(H)-1$.

Proof. For an arbitrary finite abelian group $G$ and its subgroup $K$, one can easily prove that $u(G) \leq u(G / K) u(K)$. In our situation, there exists a subgroup $K$ of $H$ such that $K \cong C_{p} \oplus C_{p}$ for some prime $p$. By Lemma 4, we have $u(K)=2 p-1 \not \leq p^{2}=\#(K)$. Thus it follows that $u(H) \leq(2 p-1) u(H / K) \not$ $\#(K) \cdot \#(H / K)=\#(H)$. Q.E.D.

From now on, we assume that $F$ is an imaginary quadratic field $K=Q(\sqrt{m})$ $(m \leq-5)$ and $F=K \notin V_{2}$.

Proposition 2. For every integer $x(0 \leq x \leq f-2)$, it is true that $x \pm w, x \pm w^{\prime}$ are irreducible elements in $K$, where $w$ is $(1+\sqrt{m}) / 2$ if $m \equiv 1(\bmod 4)$ or $=\sqrt{m}$ otherwise and $w^{\prime}$ denotes the conjugate element of $w$.

Proof. Note that $N_{K / Q}(x+y w)=(x+y / 2)^{2}-y^{2} m / 4$ if $m \equiv 1(\bmod 4)$ or $=$ $x^{2}-y^{2} m$ otherwise. Hence $y \neq 0$ implies that $N_{K / Q}(x+y w)>-m / 4$ if $m \equiv 1$ $(\bmod 4)$ or $>-m$ otherwise. Now assume that $x \pm w=\left(x_{1}+y_{1} w\right)\left(x_{2}+y_{2} w\right)$ $\left(y_{1} \neq 0, y_{2} \neq 0\right)$. Then we have $N_{K / Q}(x \pm w)>m^{2} / 16$ if $m \equiv 1(\bmod 4)$ or $>m^{2}$ otherwise. On the other hand, for every integer $x(0 \leq x \leq f-2)$, we have

$$
N_{K / Q}(x \pm w) \leq\left\{\begin{array}{r}
f^{2}-3 f+4=m^{2}+3 m+4<m^{2} \\
(m \leq-5, m \equiv 2,3(\bmod 4)) \\
f^{2}-2 f+2=m^{2} / 16+(6 m+25) / 16<m^{2} / 16 \\
(m<-5, m \equiv 1 \quad(\bmod 4)) .
\end{array}\right.
$$

Thus we have a contradiction. This completes our proof. Q.E.D.

Proposition 3. The ideal class group $H_{K}$ of $K\left(\notin V_{2}\right)$ is cyclic if and only if $n_{K}=h_{K}$.

Proof. Assume that $H_{K}=\langle x\rangle$ is cyclic. Generally, it is known that there exists an unramified prime ideal $P \in x$ with degree one (i.e., $N_{K / Q} P=p^{1}$ ) and $p \leq \sqrt{\left|d_{K}\right| / 3}<f-1$ (where $d_{K}$ is the discriminant of $K$ ). We may write $P^{h_{K}}=(a)$, where $a$ is an irreducible element in $O_{K}$. Note that $p$ is an irreducible element in $O_{K}$. (In fact, if $p=b_{1} b_{2}$ is nonunits decomposition, then it follows that $p^{2}=N_{K / Q}(p)=N_{K / Q}\left(b_{1}\right) N_{K / Q}\left(b_{2}\right)$. Hence $p=N_{K / Q}\left(b_{1}\right)$, $b_{1}=x+w y$ and $p>f-1$. This is a contradiction.) Since $a a^{\prime}=N_{K / Q}(a)=p^{h_{K}}$ and $K \in V_{n_{K}}$, we have $h_{K} \leq n_{K}$. By Proposition 1, it follows that $h_{K}=$ $n_{K}$. Conversely, assume that $h_{K}=n_{K}$ and that $H_{K}$ is not cyclic. For every irreducible element $b \in O_{K}$, we write $(b)=Q_{1} \cdots Q_{r_{b}}$ (the decomposition into prime ideals). By Lemma 5, it follows that $r_{b} \leq a\left(H_{K}\right)=u\left(H_{K}\right) \leq h_{K}-1=$ $n_{K}-1$. Hence we have $K \in V_{n_{K}-1}$ because of Lemma 2. This contradicts the definition of $n_{K}$. Therefore $H_{K}$ must be cyclic. Q.E.D.

Proposition 4. It is true that $L_{K} \leq n_{K}=a\left(H_{K}\right) \quad\left(\leq h_{K}\right)$ for $K \notin V_{2}$.

Proof. From Proposition 2 and $P_{K}(x)=(x+w)\left(x+w^{\prime}\right)$, it follows that $L_{K} \leq$ $n_{K}$. It is generally true that $K \in V_{a\left(H_{K}\right)}$ because of Lemma 2. Hence we have $n_{K} \leq a\left(H_{K}\right)$. In the case of imaginary quadratic fields, let $x_{1} \cdots x_{a\left(H_{K}\right)}=x_{0}$ be a maximal length proper relation. Since there exist unramified prime ideals $P_{i} \in x_{i} \quad\left(1 \leq i \leq a\left(H_{K}\right)\right)$ with degree one and $p_{i} \leq \sqrt{\left|d_{K}\right| / 3}<f-1$, by Lemma 
1 , it follows that $P_{1} \cdots P_{a\left(H_{K}\right)}=(c)$ for some irreducible element $c \in O_{K}$. Using the same method in the proof of Proposition 3, we know that $p_{i}$ are irreducible elements in $O_{K}$. Hence $a\left(H_{K}\right) \leq n_{K}$. Thus we have $n_{K}=a\left(H_{K}\right)$. Q.E.D.

\section{PROOF OF THE THEOREM}

Let $H^{\prime}$ be a finite abelian group such that $\#\left(H^{\prime}\right)=h_{K} \quad\left(=\#\left(H_{K}\right)\right)$. Suppose that $H^{\prime} \cong C_{b_{1}} \oplus \cdots \oplus C_{b_{r}}, b_{i} \mid b_{i+1} \quad(1 \leq i \leq r-1), 2 \leq r, 2 \leq b_{1}, h_{K}=$ $b_{1} \cdots b_{r}$. Since $q_{0} b_{1} \leq b_{j} b_{1} \leq h_{K}$ for some $j(1 \leq j \leq r-1)$, it follows that $b_{1}-q_{0} \leq h_{K} / q_{0}-h_{k} / b_{1}$. Hence we have $b_{1}+h_{K} / b_{1}-1 \leq q_{0}+h_{K} / q_{0}-1=$ $u\left(C_{q_{0}} \oplus C_{h_{K} / q_{0}}\right)$ because of Lemma 4 . Now define a real-valued differentiable function $f(y, z)=y z-\log (y z)-\log y$ with two variables $y, z \quad(2 \leq y, 2 \leq z)$. Then $\partial f / \partial z=y-1 / z=0, \partial f / \partial y=z-2 / y=0$ has no common root. Since $f(y, z) \geq 4-\log 4-\log 2 \nsupseteq 4-2.08 \nsupseteq 1$, we have $b_{2} \cdots b_{r-1}-\log \left(b_{2} \cdots b_{r-1}\right)-$ $\log b_{1} \nsucceq 1$. Therefore we have

$$
\begin{aligned}
b_{r}-b_{1}+1 & \not \leq b_{r} \\
& =h_{K} / b_{1}-b_{r}\left\{b_{2} \cdots b_{r-1}-\log \left(h_{K} / b_{r}\right) .\right.
\end{aligned}
$$

This shows that

$$
b_{r}\left\{1+\log \left(h_{K} / b_{r}\right)\right\} \not b_{1}+h_{K} / b_{1}-1 \leq q_{0}+h_{K} / q_{0}-1 .
$$

The above argument says that

$\max \left\{u\left(H^{\prime}\right) ; \#\left(H^{\prime}\right)=h_{K}\right.$ and $H^{\prime}$ is noncyclic abelian $\}=q_{0}+h_{K} / q_{0}-1$.

We already know that $L_{K} \leq n_{K}=a\left(H_{K}\right)=u\left(H_{K}\right) \leq h_{K}$. Therefore, $L_{K} \geq$ $q_{0}+h_{K} / q_{0}$ implies that $n_{K}=h_{K}$. Thus $H_{K}$ is cyclic because of Proposition 3. Q.E.D.

\section{NUMERICAL EXAMPLES}

Since our sufficient condition is not available for $L_{K}$ smaller than $\sqrt{h_{K}}$, we have only 'limited' applications. However there are some examples for application of our theorem as follows.

In Table 1 , the group $H_{K}$ is cyclic by our theorem. For example, let $m=$ -199 ; then $f=(1-m) / 4=50$. For the polynomial $P_{K}(x)=x^{2}+x+50(0 \leq$ $x \leq 50-2)$, we have $P_{K}(21)=512=2^{9}$. Hence $9 \leq L_{K} \quad(K=Q(\sqrt{-199}))$. On the other hand, it is known that $L_{K} \leq h_{K}=9$. Therefore $L_{K}=h_{K}=9$.

TABLE 1. There are some examples such that $L_{K}=h_{K}$ and $h_{K}$ has a square factor.

$$
-m
$$

$14,17,34,39,55,97,142,155,203,219,259$,

$291,323,667,955,1027,1227,1411,1507$

$183,295,583$

$199,367,1087,1423$
$L_{K} \quad h_{K}$

44

44

$8 \quad 8$

$9 \quad 9$

$12 \quad 12$

$16 \quad 16$




\section{TABLE 2}

$\begin{array}{rcc}-m & L_{K} & h_{K} \\ 419 & 7 & 9 \\ 491 & 6 & 9 \\ 563 & 6 & 9 \\ 1187 & 6 & 9\end{array}$

TABLE 4

$\begin{array}{ccc}-m & L_{K} & h_{K} \\ 362 & 9 & 18 \\ 519 & 10 & 18 \\ 527 & 9 & 18 \\ 679 & 9 & 18 \\ 1135 & 11 & 18 \\ 1142 & 9 & 18 \\ 1207 & 10 & 18 \\ 1373 & 9 & 18 \\ 1383 & 10 & 18 \\ 1687 & 9 & 18 \\ 1927 & 10 & 18 \\ 2047 & 10 & 18 \\ 2167 & 12 & 18 \\ 3035 & 10 & 18 \\ 3107 & 9 & 18 \\ 3543 & 14 & 18 \\ 7571 & 9 & 18 \\ 7715 & 9 & 18\end{array}$

TABLE 3

$\begin{array}{rrr}-m & L_{K} & h_{K} \\ 479 & 10 & 25 \\ 599 & 11 & 25 \\ 1367 & 13 & 25 \\ 2887 & 12 & 25 \\ 5503 & 12 & 25 \\ 7187 & 10 & 25 \\ 7287 & 11 & 25 \\ 4591 & 16 & 49 \\ 7879 & 15 & 49\end{array}$

\section{TABLE 5}

$-m \quad L_{K} \quad h_{K}$

$\begin{array}{lll}1231 & 12 & 27\end{array}$

$\begin{array}{lll}1399 & 13 & 27\end{array}$

$\begin{array}{lll}3943 & 13 & 27\end{array}$

$\begin{array}{lll}4903 & 12 & 27\end{array}$

$\begin{array}{lll}6007 & 12 & 27\end{array}$

In Tables 2 and 3, we have $L_{K} \geq 3+9 / 3=6,5+25 / 5=10,7+49 / 7=14$, respectively. Hence $H_{K}$ is cyclic by our theorem.

In Tables 4 and 5 , we have $L_{K} \geq 3+18 / 3=9,3+27 / 3=12$, respectively. Hence the ideal class group $H_{K}$ of $K=Q(\sqrt{m})$ is cyclic by our theorem.

Remark. Tables 1-5 were extracted from the unpublished numerical tables on imaginary quadratic number fields $K=Q(\sqrt{m})(-8187<m<0)$ by H. Wada (1985).

\section{REFERENCES}

1. P. C. Baayen, P. Emde Boas, and D. Kruyswijk, A combinatorial problem of finite abelian groups. III, ZW 1969-008, Report Math. Centrum, Amsterdam, 1969.

2. L. Carlitz, A characterization of algebraic number fields with class number two, Proc. Amer. Math. Soc. 11 (1960), 391-392. 
3. A. Czogala, Arithmetical characterization of algebraic number fields with small class number, Math. Z. 176 (1981), 247-253.

4. W. Narkiewicz, Finite abelian groups and factorization problems, Colloq. Math. 42 (1979), 319-330.

5. J. E. Olson, A combinatorial problem on finite abelian groups. II, J. Number Theory 1 (1969), 195-199.

6. G. Rabinovitch, Eindeutigkeit der zerlegung in primzahlfaktoren in quadratischen zahlkörpern, Proc. Fifth Internat. Congr. Math., vol. I, 1912, pp. 418-421=J. Reine Angew. Math. 142 (1913), 153-164.

7. R. Sasaki, On a lower bound for the class number of an imaginary quadratic field, Proc. Japan Acad. Ser. A Math. Sci. 62 (1986), 37-39.

IwaKi Meisei University, 1-27-10 Kitahara-cho, TANAShi-ShI, Tokyo 188, Japan 